\title{
Stellar populations associated with the LMC Papillon Nebula ${ }^{\star \star \star}$
}

\author{
F. Meynadier ${ }^{1}$, M. Heydari-Malayeri ${ }^{1}$, L. Deharveng ${ }^{2}$, V. Charmandaris ${ }^{3,1}$, Th. Le Bertre ${ }^{1}$, \\ M. R. Rosa ${ }^{4, \star \star \star}$, D. Schaerer ${ }^{5,6}$, and H. Zinnecker ${ }^{7}$
}

\author{
1 LERMA, Observatoire de Paris, 61 avenue de l'Observatoire, 75014 Paris, France \\ 2 Observatoire de Marseille, 2 place Le Verrier, 13248 Marseille Cedex 4, France \\ 3 Cornell University, Astronomy Department, 106 Space Sciences Bldg., Ithaca, NY 14853, USA \\ ${ }^{4}$ Space Telescope European Coordinating Facility, European Southern Observatory, Karl-Schwarzschild-Strasse-2, \\ 85748 Garching bei München, Germany \\ 5 Observatoire de Genève, 51 Ch. des Maillettes, 1290 Sauverny, Switzerland \\ ${ }^{6}$ Laboratoire d'Astrophysique, UMR 5572, Observatoire Midi-Pyrénées, 14 avenue E. Belin, 31400 Toulouse, France \\ 7 Astrophysikalisches Institut Potsdam, An der Sternwarte 16, 14482 Potsdam, Germany
}

Received 16 December 2003 / Accepted 22 March 2004

\begin{abstract}
We study the Large Magellanic Cloud Papillon Nebula (N 159-5), a conspicuous High Excitation Blob (HEB) lying in the star forming complex N 159. Using $J H K$ near-infrared photometry obtained at the ESO VLT with the ISAAC camera, we examine the stellar populations associated with the Papillon, tracing their history using stellar evolution models. Two populations are revealed: one composed of young, massive stars with an age $\sim 3 \mathrm{Myr}$, and a second consisting of older lower mass stars of age spreading between 1 and $10 \mathrm{Gyr}$. We analyze the properties of those populations and discuss their significance in the context of N 159. We also estimate that if the star at the center of the Papillon is single its initial mass is $\sim 50 M_{\odot}$ and it is affected by an extinction $A_{\mathrm{V}} \sim 7$ mag.
\end{abstract}

Key words. stars: early-type - ISM: individual objects: N 159 LMC - galaxies: Magellanic Clouds

\section{Introduction}

The compact H II regions called High-Excitation Blobs (HEB) constitute a rare class of ionized nebulae in the Magellanic Clouds. They are characterized by high excitation, small size, high density, and large extinction compared to typical Magellanic Cloud H II regions. These objects are tightly linked to the early stages of massive star formation, when the stars begin to hatch from their parental molecular clouds. For this reason their study yields valuable information for a better understanding of the formation of massive stars.

The N 159 complex (Henize 1956) lies south of the famous starburst site 30 Dor and has attracted special attention over the years. Its other designations are MC 77 (McGee et al. 1972), LH 105 (Lucke \& Hodge 1970), and DEM 271 (Davies et al. 1976). It shows several signs of ongoing star formation activity, such as infrared sources, cocoon stars, masers, and is also

Send offprint requests to: $\mathrm{F}$. Meynadier,

e-mail: Frederic. Meynadier@obspm. fr

* Based on observations obtained at the European Southern Observatory, Paranal, Chile; Program 66.C-0172(A).

$\star \star$ Table 1 is only available in electronic form at the CDS via anonymous ftp to cdsarc.u-strasbg.fr $(130.79 .128 .5)$ or via http://cdsweb.u-strasbg.fr/cgi-bin/qcat?J/A+A/422/129 $\star \star \star$ Affiliated to the Space Telescope Division of the European Space Agency, ESTEC, Noordwijk, The Netherlands. associated with the most important concentration of molecular gas in the LMC (Jones et al. 1986; Brooks \& Whiteoak 1997; Johansson et al. 1998). The molecular emission is in fact due to three distinct giant molecular clouds, known as N159-East, N159-West, and N159-South. Molecular lines tracing high density regions are observed towards $\mathrm{N} 159-\mathrm{W}$ and $\mathrm{N} 159-\mathrm{S}$ in CS, $\mathrm{CN}, \mathrm{HCN}$, and $\mathrm{HCO}^{+}$(Heikkilä et al. 1999), while ${ }^{13} \mathrm{CO}$ and upper-level ${ }^{12} \mathrm{CO}$ transitions, and the [C II] emission line were mapped towards the three giant molecular clouds, including $\mathrm{N} 159$-E (Bolatto et al. 2000). The region we are interested in lies near N 159-E.

The Papillon Nebula, also called N 159-5, to which the present study is devoted, is the prototype of the HEB family (Heydari-Malayeri \& Testor 1982), which now possesses several members, such as N 160A1, N 160A2, N 83B, N 11A in the LMC and N 88A and N 81 in the SMC. Recent HST observations have resolved most of these objects and revealed turbulent media typical of newborn massive star regions marked by strong stellar winds interacting with the ambient ionized gas (Heydari-Malayeri et al. 1999a,b,c, 2001a,b, 2002a,b). These observations also showed a large extinction due to local dust associated with ionized gas. In a number of cases the exciting sources were uncovered as a small cluster of massive stars.

In particular, the WFPC2 HST observations revealed that the featureless blob N 159-5 has in fact the morphology of a 
"papillon", i.e. it is a butterfly-shaped ionized nebula with the "wings" separated by 2".3 (0.6 pc) (Heydari-Malayeri et al. 1999b, hereafter Paper I). Moreover, two subarcsecond features resembling a "smoke ring" and a "globule" were detected in the wings. The images also showed a large number of subarcsecond filaments, arcs, ridges, and fronts carved in the ionized gas by the stellar winds from massive stars in the N 159 complex. However, no bright stars associated with the core of N 159-5 could be identified in the HST images. Could this be due to the extinction by dust large enough to hide the exciting stars? Since an $A_{\mathrm{V}} \geq 6$ mag was needed to bring an 08 star below our HST sensitivity limit, we decided to perform high resolution near-IR imaging of the region with the ESO Very Large Telescope in order to address this issue and explore the properties of the stellar population of N 159.

\section{Observations and data reduction}

The N 159 region was observed in service mode with the ESO Very Large Telescope (VLT). The infrared spectro-imager ISAAC was used at the Nasmyth B focus of UT1 through filters $J$ on 7 October and $H$ and $K$ s on 1 st March 2001. The IR detector (Hawaii Rockwell array) had $1024 \times 1024$ pixels of $18.5 \mu \mathrm{m}$ each $\left(0{ }^{\prime} 148\right.$ on the sky), thus providing a field of $2.5^{2}$.

A set of individual, 10-s exposures was obtained in each filter using the dithering method with a random offset of $15^{\prime \prime}$ at most. The number of exposures were 10, 30, and 36 for the $J$, $H$, and $K$ s bands respectively. The coadded frames have a spatial resolution of 1'.'08 for $J, 0$.'74 for $H$, and $0 . ' 63$ for $K$ s.

PSF-fitting photometry was carried out in the $J, H$, and $K$ s filters using the DAOPHOT II/ALLSTAR procedures (Stetson 1987) under the ESO MIDAS reduction package. It should be noted that these procedures are well adapted to the high-precision photometry of globular clusters (i.e. tight groups of point sources with no background emission), but are not designed for handling regions with very bright and variable background such as N 159. Some alternatives to address those limitations have been investigated by Deharveng et al. (1992), and involve an iterative subtraction of the background as derived from the approximative photometry obtained at each step. In order to improve this method, we developed a software, called DENEB for DE-NEBulized photometry. Our software enables an interactive modification of the intermediate photometry files as well as a real time check of the validity and convergence of those modifications since it displays the resulting residual background ${ }^{1}$.

Finally the frames were calibrated using the mean atmospheric extinction coefficients and the color equations supplied by ESO, and three standard stars for determining the zero points. The average photometric errors reported by DAOPHOT are $0.04,0.04$, and $0.05 \mathrm{mag}$ for the faintest stars in $J, H$, and $K$ s respectively. The relative accuracy is better than 0.01 mag for $J, H$, and $K$ s brighter than 17 mag.

\footnotetext{
${ }^{1}$ People interested in the use or development of this tool are invited to contact Frederic . Meynadier@obspm . fr
}

We compared the resulting magnitudes with those provided by the 2MASS point source catalogue (Cutri et al. 2003a) using a selection of 36 stars which appeared as single in our frames and were brighter than $15.0 \mathrm{mag}$ in $H$. After correction for filter bandpasses (Carpenter 2001; Cutri et al. 2003b) the mean differences are $m(2 \mathrm{MASS})-m($ ISAAC $)=-0.06$, -0.08 , and $-0.02 \mathrm{mag}$ in $J, H$, and $K$ s respectively. Taking into account the accuracy of the 2MASS photometry for stars of $H=15 \mathrm{mag}(\sim \pm 0.1 \mathrm{mag} \mathrm{rms})$ and the uncertainties involved in the filter bandpass corrections, we considered these mean differences not to be significant.

Our astrometry and image registration was tied to the positions of the same 2MASS stars since it is known that the rms uncertainty in the positions of the 2MASS catalogue is $<0{ }^{\prime} 3$. The astrometry and the photometry of the stars are given in Table 1, which is available in electronic form at the CDS.

\section{Results}

\subsection{Morphology}

A composite color $J H K$ s image of the observed field is shown in Fig. 1, while the corresponding finding chart is presented in Fig. 2. The stars are identified by a number, according to Table 1. Figures 3 and 4 give details on two densely populated regions, the central Papillon nebula and a small southern cluster.

The field is fairly rich, with 896 stars detected at a $3 \sigma$ level in the $H$ band image (limiting magnitude 20), which has the best $S / N$ ratio. Among them 605 objects are detected at $3 \sigma$ on all theses frames. Some particularly bright, but highly reddened stars do not appear in all three filters and consequently they were not included in the analysis. Exception was made for a source labeled as \#317.1 which is not detected in the $J$ band while being relatively bright in $K \mathrm{~s}$.

The image is marked by several dark regions and lanes indicating strong absorption. The Papillon nebula is situated near the border of a prominent central absorption lane. The background is dominated by ionized gas emission but is locally obscured by heavy extinction. The southern edge of the field yields a fan-shaped filament already visible on the HST frames (Paper I). Our new JHKs imagery provides a deeper overview of the stellar content of $\mathrm{N} 159$. We can easily note that the small, bright cluster south to the Papillon (area B of Fig. 2) is much more visible in the near IR than in the optical (Paper I). The two reddest stars of the field are \#210 and \#317.1. The first one is located in the lower right quadrant of our images almost 1 arcmin from $\mathrm{N} 159-5$, and the second one near the edge of the absorption lane.

We also note the presence of a number of "peculiar" objects having an elongated form and a red color: \#343, \#517, and \#149. The first one seems also to have a tilted shape. The probability that they result from a chance alignment of several faint red stars is very low. Since they are physically too extended to be considered as circumstellar disks of the LMC, it is more likely that they are simply background galaxies. 


\subsection{The infrared colors and stellar ages}

Figure 5 presents the $K$ s versus $H$-Ks diagram for the measured stars towards N 159, while Fig. 6 displays the corresponding color-color $J-H$ versus $H-K$ s diagram. All sources brighter than $H=20$ mag and also detected in $J$ and $K$ s are taken into account. Star \#317.1, which is not detected in $J$, is assigned an upper limit of 20 in this band. We wish to point several stars in our sample which display rather unique properties. The square in Figs. 5 and 6, identifies star \#371, which is the central point-like source of the Papillon. The eight triangles correspond to the brightest components of the small southern cluster marked as region B in Fig. 2.

The color-magnitude and color-color diagrams are interpreted by overplotting isochrones from Lejeune \& Schaerer (2001) with $Z=0.008$ for a distance modulus of $18.5 \mathrm{mag}$. As usual with near-IR observations, it is difficult to discriminate low-mass old stars from young massive stars, because the evolutionary tracks are nearly parallel to isochrones, resulting in a very close location on the color-color diagram for those two populations. This degeneracy is lifted if the mass is taken into account: color-magnitude and color-color diagrams should be "coherent", in the sense that populations found to be fitted by a given isochrone in one diagram should be fitted by the same isochrone, within the same mass interval, in the second. Uncertainties in the photometry though, as well as lack of knowledge in the variation of the extinction introduce limitations to the precise determination of the corresponding isochrone.

The diagrams show the presence of two stellar populations. The first one is a young population which appears to be fitted well with a $3 \mathrm{Myr}$ isochrone. Some of the members of this population are weakly affected by extinction while other members have reddened colors. The extinction-free subset is vertically aligned around $H-K \mathrm{~s}=0.00$ in Fig. 5, and the sample affected by extinction has $H-K$ s colors around 0.20 mag. This young population is made up of massive $\mathrm{O}$ type stars, and may also contain a component of reddened B type stars of $\sim 15 M_{\odot}$ spread around $H-K \mathrm{~s}=0.2 \mathrm{mag}$. Apart from this young population, there is a second population with generally redder colors, which can be fitted with much older isochrones of least 1 to $10 \mathrm{Gyr}$ in age. The bulk of the stars in this population are fainter than $K \mathrm{~s}=17 \mathrm{mag}$ and have a mass of about $1 M_{\odot}$, although the brightest members have evolved into giants. This population is also affected by a varying amount of extinction. The points lying to the right of the 1-10 Gyr isochrones are much more extincted, probably representing the stars situated deeper in the molecular cloud. As was mentioned earlier, it is not clear which precise isochrone should be used, because we expect the extinction to be generally high and locally variable. It is, however, evident that this second population is significantly older than the first one, and we can notice the existence of a considerable spread in age among this population. It should also be underlined that for the metallicity of the LMC a star of initial mass $2.15 M_{\odot}$ evolves into a giant in less than $\sim 1 \mathrm{Gyr}$.

The color-magnitude diagram can be used to estimate the extinction of the stars. Assuming that the triangles represent young massive stars of age $\sim 3 \mathrm{Myr}$, their shift to the right in
Fig. 5 is attributed to reddening by dust. An extinction of $A_{\mathrm{V}} \sim$ $5 \mathrm{mag}$ is sufficient to displace the mean position of that stellar population. The star \#371, detected towards the Papillon, does not seem to have an IR excess, but is affected by an extinction of $A_{\mathrm{V}} \sim 7 \mathrm{mag}$. This is consistent with Paper I, which found $A_{\mathrm{V}} \geq 6$ for this central region on the assumption that extinction would have to be large enough to hide an hypothetical O8 exciting star. See Sect. 3.3 for comparison with the CO map.

\subsubsection{Isochrone fitting}

The apparent magnitude of star \#193, better known as R 149 or Sk-69 257 , is $V=12.389$ with $B-V=-0.081, U-B=$ -0.962 mag (Schmidt-Kaler et al. 1999), in agreement with $V=12.49$ mag (Dufour \& Duval 1975), and corresponds to an absolute magnitude of $M_{V} \sim-6.8$ to -6.6 . For an O9 spectral type (Walborn 1977; Conti et al. 1986) this is marginally consistent with luminosity class III, but typical of class I (Vacca et al. 1996). A dwarf classification, as suggested by Conti et al. (1986), seems therefore excluded. The effective temperature of O9 III-I stars is $T_{\text {eff }} \sim 34300-32700 \mathrm{~K}$ using Vacca et al. (1996)'s calibration or typically between 31.6 and $27500 \mathrm{~K}$ for the extreme Ia class based on recent model analysis taking into account non-LTE line blanketing (Martins et al. 2002; Crowther et al. 2002; Herrero et al. 2002; Markova et al. 2004). The bolometric correction, which is essentially independent of line blanketing, is then $B C \sim-3.4$ to -2.7 (Vacca et al. 1996) translating to luminosities $\log L / L_{\odot} \sim 5.6-6.0$.

Figure 7 presents the Geneva evolutionary tracks calculated for initial masses $20,25,40$, and $60 M_{\odot}$ and metallicities $Z=$ 0.008 and 0.02 (Lejeune \& Schaerer 2001). We reported the position of R 149 on this diagram, using the values calculated in the previous paragraph. It indicates an initial mass of the order of 40 to $60 M_{\odot}$, which corresponds to an age of 3 to $5 \mathrm{Myr}$ using the initial masses predicted for stars of various ages and $M_{V}$ between -6.8 and -6.6 mag (Fig. 8, top). This is consistent with the age range, 1 to $4 \mathrm{Myr}$, derived from the observed $K$ s magnitude (Fig. 8, bottom).

\subsubsection{The brightest and reddest stars}

Based on the color-magnitude and color-color diagrams (Figs. 5 and 6), stars \#588, \#290, \#499, \#404, \#365, \#77, and \#210 may be high mass main sequence members. These stars can also be very tight multiple systems more or less affected by local dust. In particular, \#365 and \#210 are very red, probably due to their association with the prominent absorption features in Fig. 1. Furthermore, star \#210 presents a near infrared excess typical of some Galactic OB exciting stars, for example star \#82 ionizing the H II region Sh2-88B (Deharveng et al. 2000). It is not easy to estimate the extinction and the mass of such a star, more especially since the possible presence of a circumstellar disk alters the colors (Lada \& Adams 1992). If single, this star would be one of the most massive stars of the region, having a mass of $\sim 100 M_{\odot}$ while affected by an $A_{\mathrm{V}}>$ $20 \mathrm{mag}$. 


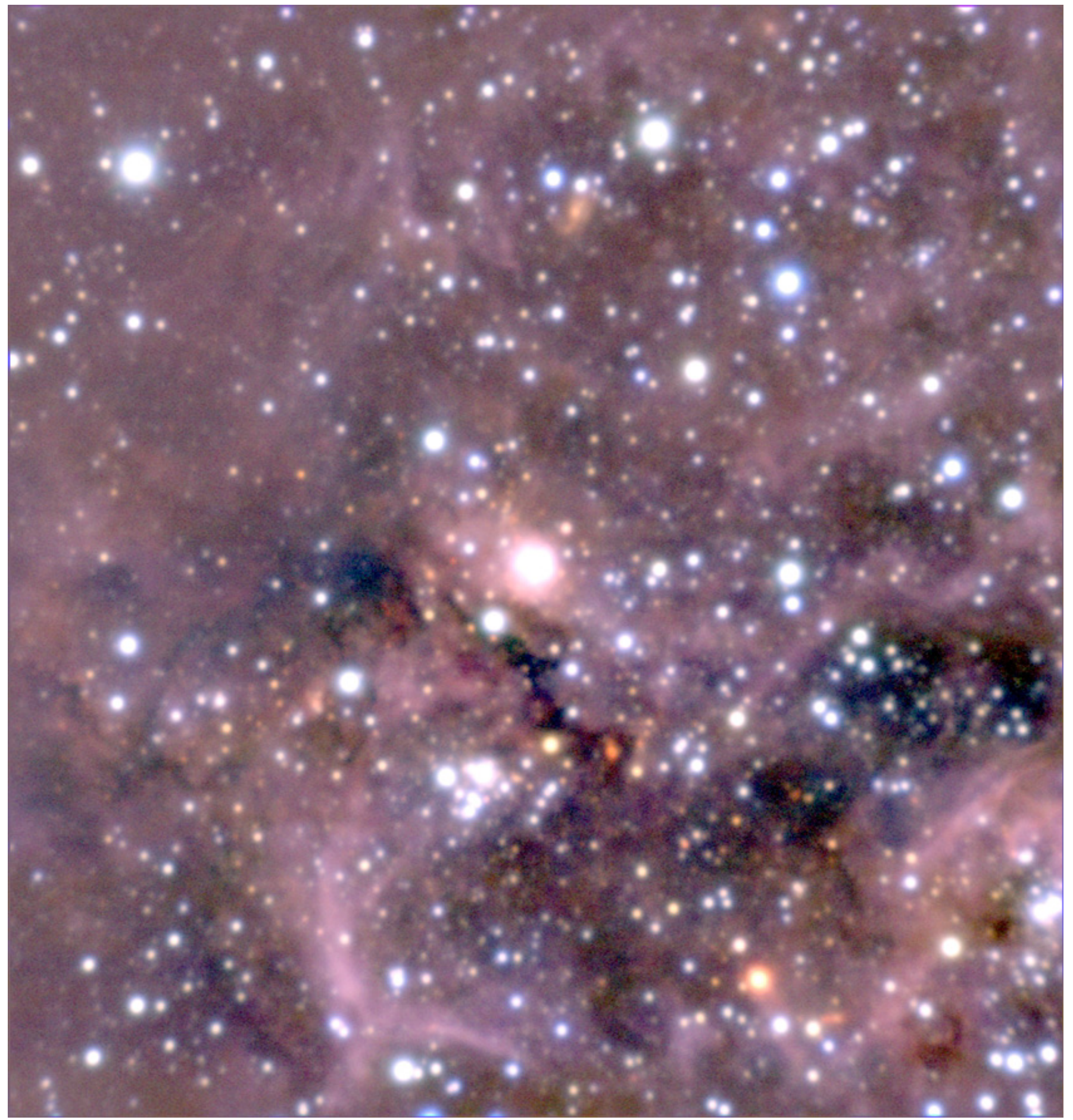

Fig. 1. $J H K$ color composite image of LMC N $159(K \mathrm{~s}=$ red, $H=$ green, $J=$ blue) centered on N 159-5, the Papillon nebula. North is up and East is left. See Fig. 2 for star identifications. The field size is 2 ' $1 \times 2$ '.2 $(32 \mathrm{pc} \times 33 \mathrm{pc})$.

A number of reasons also suggest that some of these stars may be LMC supergiants. Using a bolometric correction of +2.7 mag in the $K$ band for supergiant stars (van Loon et al. 1999; Le Bertre et al. 2001) and an extinction of $A_{K} \sim 0.5$ or $1.0 \mathrm{mag}$, we find that the brightest stars of the sample have an absolute $M_{K}$ and bolometric $M_{\mathrm{b}}$ magnitudes in the range -8 to -8.5 and -5.7 to -6.2 respectively. These magnitudes are consistent with $\mathrm{M}$ type supergiants, carbon stars, or fainter AGB stars in the LMC (van Loon et al. 1999; van Loon 2000).
As for \#210, which has a redder color of $H-K \mathrm{~s}=2.1 \mathrm{mag}$, it can qualify as an LMC AGB candidate. Future spectroscopic observations are needed in order to clarify the nature of these stars.

A third possibility is that at least some of these stars actually belong to our Galaxy and happen to be along the line of sight to the LMC. We can make a rough estimate on their number by establishing the H-R diagram of 2MASS sources found in a field separated by a few degrees from the LMC. 


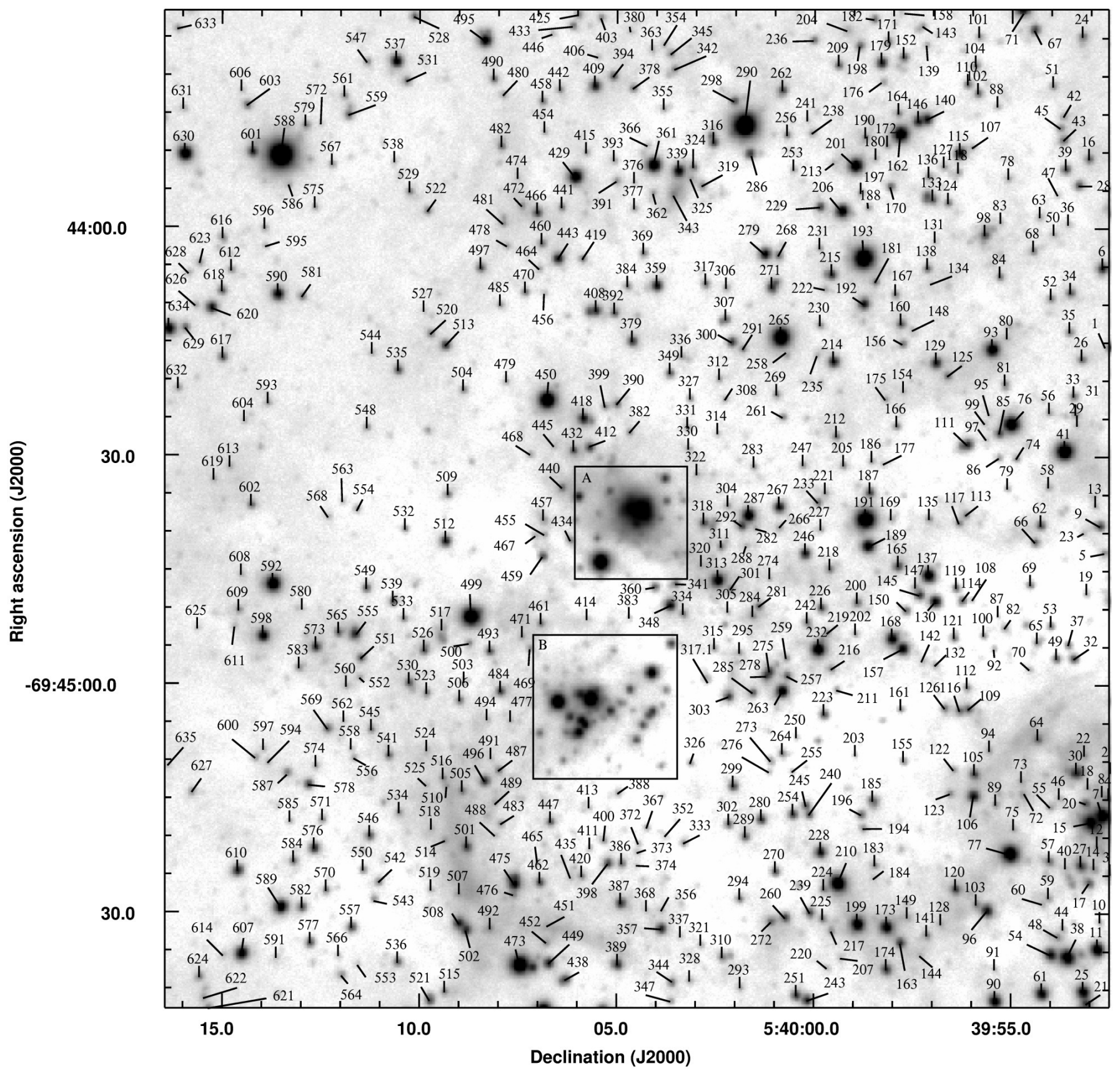

Fig. 2. Finding chart ( $H$ filter) for the stars in Fig. 1. The numbers refer to Table 1. Regions A (N 159-5) and B are detailed below.
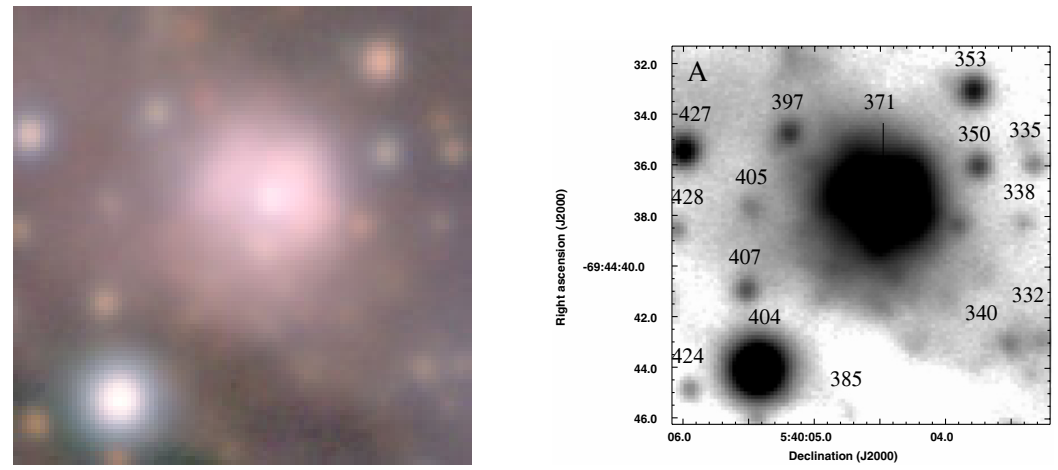

Fig. 3. Composite $J H K$ s image $(K \mathrm{~s}=$ red, $H=$ green, $J=$ blue $)$ and the corresponding finding chart ( $H$ band) for region A, the central Papillon (see Paper I).

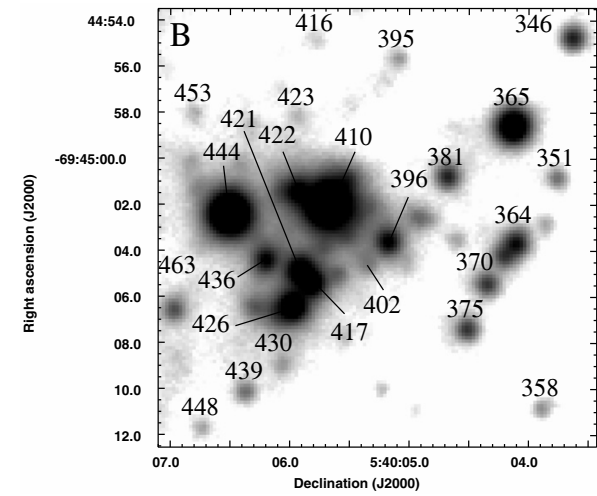

Fig. 4. Finding chart for region B ( $H$ band). 


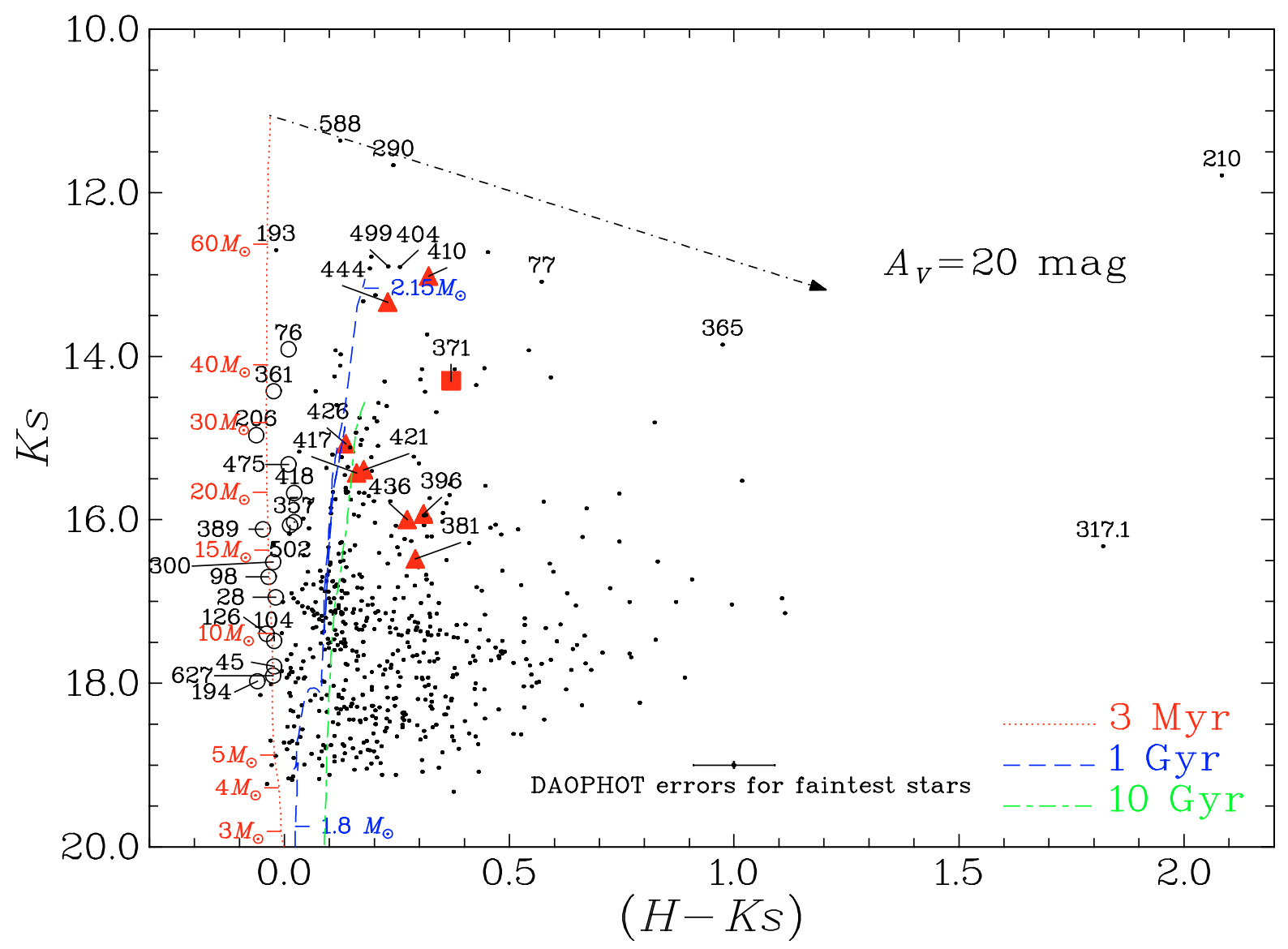

Fig. 5. Color-magnitude, $K$ s versus $H-K$ s, diagram for the observed stars towards LMC N 159 detected in all three filters. Three isochrones are shown, $3 \mathrm{Myr}$ (dotted red curve), $1 \mathrm{Gyr}$ (dashed blue), and $10 \mathrm{Gyr}$ (dashed-dotted green), computed for a metallicity of $Z=0.008$ (Lejeune \& Schaerer 2001) and a distance modulus of 18.5 mag. The upper and lower mass limits are indicated for the 1 Gyr isochrone. The reddening track, plotted as an arrow, extends to $A_{\mathrm{V}}=20 \mathrm{mag}$. The numbers refer to the stellar identifications presented in Fig. 2. Triangles represent a sample of the stars belonging to region B, while the square (numbered \#371) refers to the central point-like source of the Papillon. Stars brighter than $K \mathrm{~s}=18 \mathrm{mag}$ and situated near the $3 \mathrm{Myr}$ isochrone are labelled and shown as empty circles.

It appears that in our field $\sim 14$ sources brighter than $K \mathrm{~s}=$ 15 mag might be foreground stars. Those stars cannot be compared to models computed for the LMC distance modulus. Our observations also indicate that there are 45 stars brighter than $K \mathrm{~s}=15$, so approximately 30 of them should be considered as belonging to the LMC. The above-mentioned bright stars have colors placing them in the low-mass end of any isochrones between 1 to 10 Gyr (Fig. 6) adapted to the LMC, even though they are among the most luminous sources in Fig. 5 where they are located near the high-mass end of the same isochrones. This apparent contradiction can be explained if they are foreground Galactic stars: their location should be compared to "shifted" isochrones in the color-magnitude diagram in order to account for their different distance moduli, while the color-color isochrones would remain unchanged.

\subsection{The molecular gas distribution}

Johansson et al. (1998) used the ESO SEST (Swedish European Submillimeter Telescope) to map the CO (1-0) emission towards N 159 with a resolution of 40". We performed a bilinear interpolation between each grid point in order to generate the contours corresponding to the molecular gas associated with the Papillon region. The result, representing the $\mathrm{CO}$ emission peak called N 159-East, is overlayed on the $H$ image and presented in Fig. 9.

The constraints on $A_{\mathrm{V}}$ established in Paper I and in the present work are in good agreement with Fig. 9. Since the mapping is relatively scarce, the location of the two peaks cannot be precisely determined but they coincide with the absorption feature bordering the compact $\mathrm{H}$ II region. The present picture is in perfect agreement with previous findings that the main $\mathrm{CO}$ peak is shifted to the east of the bulk of the giant $\mathrm{H}$ II region N 159 mapped in the radio continuum at $843 \mathrm{GHz}$ (Mills \& Turtle 1984; Heydari-Malayeri \& Testor 1985; Israel et al. 1996). Regions A and B are both adjacent to the molecular peaks, region $\mathrm{B}$ being less affected by extinction. It is conceivable that more massive stars be in the process of birth towards the $\mathrm{CO}$ emission maxima.

In order to estimate the extinction corresponding to the $\mathrm{CO}$ peak the optically thin ${ }^{13} \mathrm{CO}$ transition is needed. Bolatto et al. (2000) observed the N $159-\mathrm{W}$ component in ${ }^{13} \mathrm{CO}$ and derived a column density of $1.1 \times 10^{22} \mathrm{~cm}^{-2}$ for the molecular hydrogen $\mathrm{H}_{2}$, corresponding to a column density of 


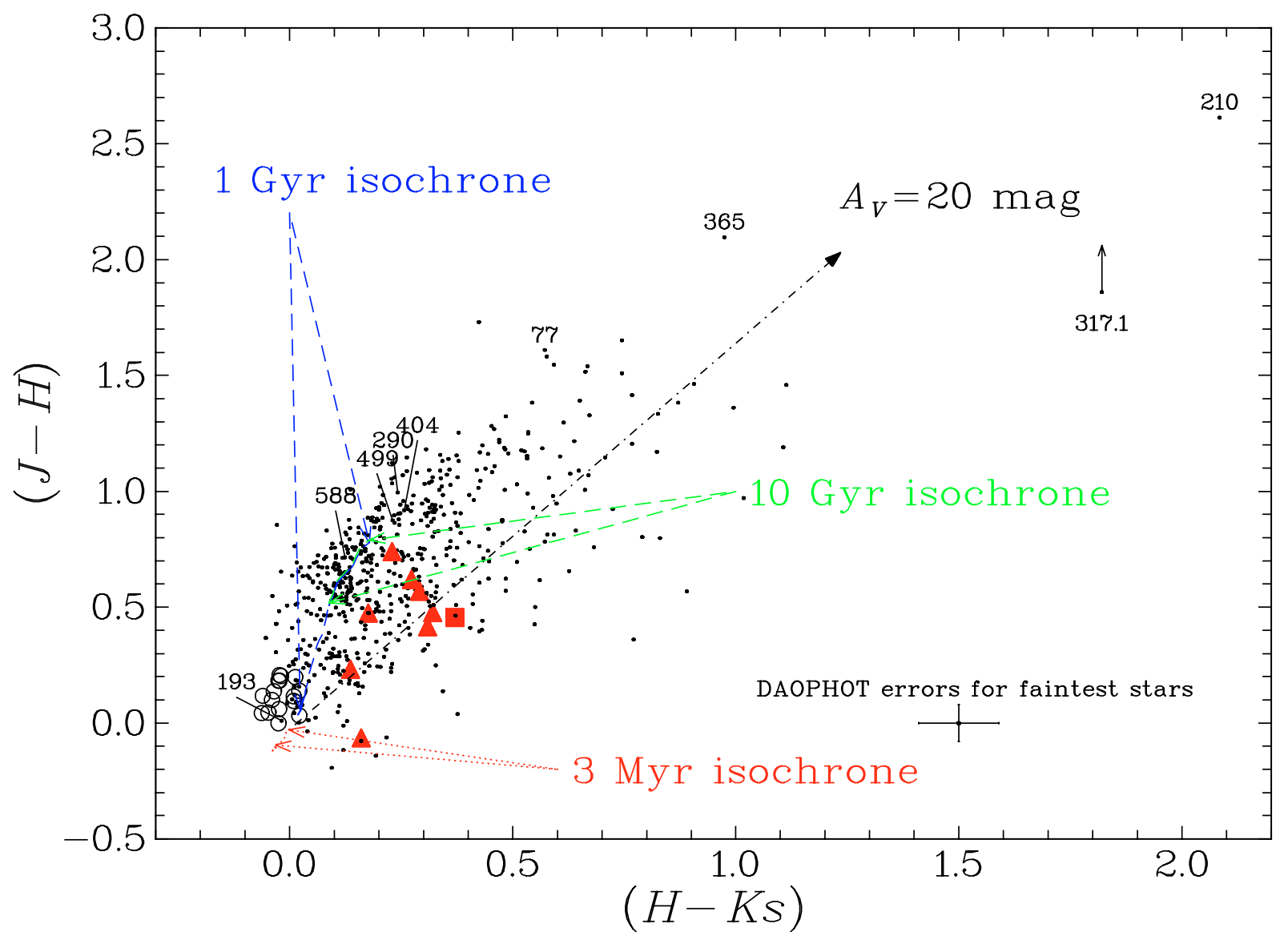

Fig. 6. Color-color, $J-H$ versus $H-K$ s, diagram for the observed LMC N 159 stars. The isochrone reference, symbols, and the reddening track are as in Fig. 5. The arrow attached to star \#317.1 is due to the fact that an upper limit of 20 in the $J$ band has been assumed for it.

atomic hydrogen of $2.2 \times 10^{22} \mathrm{~cm}^{-2}$. It is known that the gas-to-dust ratio $N_{\mathrm{H}} / E(B-V)$ in the LMC is several times larger than the Galactic value (Nandy et al. 1981; Clayton \& Martin 1985; Lequeux 1989). Using the conversion relation $N_{\mathrm{H}} / E(B-V)=2 \times 10^{22}$ atoms $\mathrm{cm}^{-2} \mathrm{mag}^{-1}$ given by Lequeux (1989) and $R=A_{\mathrm{V}} / E(B-V)=3.1$, we find a visual extinction of $A_{\mathrm{V}} \sim 3.5 \mathrm{mag}$ for $\mathrm{N} 159-\mathrm{W}$. The extinction for N 159-E should be smaller since we know that N 159-E is less dense than N159-W (see below). On the other hand, Dickey et al. (1994) carried out 21-cm H I absorption line observations against background continuum sources towards N 159 using the Australia Telescope Compact Array (ATCA) interferometer. Their H I cloud 0539-697 can be identified with the molecular cloud N 159-E, based on velocity similarity (Johansson et al. 1998). The CO cloud has the following characteristics: $V=238 \mathrm{~km} \mathrm{~s}^{-1}, \Delta V=6.0 \mathrm{~km} \mathrm{~s}^{-1}, \log \left(L_{\mathrm{CO}}\right)=$ $4.28 \mathrm{~K} \mathrm{~km} \mathrm{~s}^{-1} \mathrm{pc}^{2}$, whereas those for the H I cloud are: $V=$ $244 \mathrm{~km} \mathrm{~s}^{-1}, \Delta V=1.6 \mathrm{~km} \mathrm{~s}^{-1}, N(\mathrm{H} \mathrm{I})=4.46 \times 10^{22} \mathrm{~cm}^{2}$. This column density corresponds to a visual extinction of $A_{\mathrm{V}} \sim$ $7 \mathrm{mag}$ while that for N $159-\mathrm{W}$, i.e. $N(\mathrm{H} \mathrm{I})=9.62 \times 10^{22} \mathrm{~cm}^{-2}$, indicates a larger extinction of $A_{\mathrm{V}} \sim 15 \mathrm{mag}$. A reason why $\mathrm{HI}$ observations yield stronger extinctions is that the higher spatial resolution of the interferometer picks up denser clumps, in contrast to $\mathrm{CO}$ observations which are affected by beam dilution. Moreover, it is quite possible that both techniques do

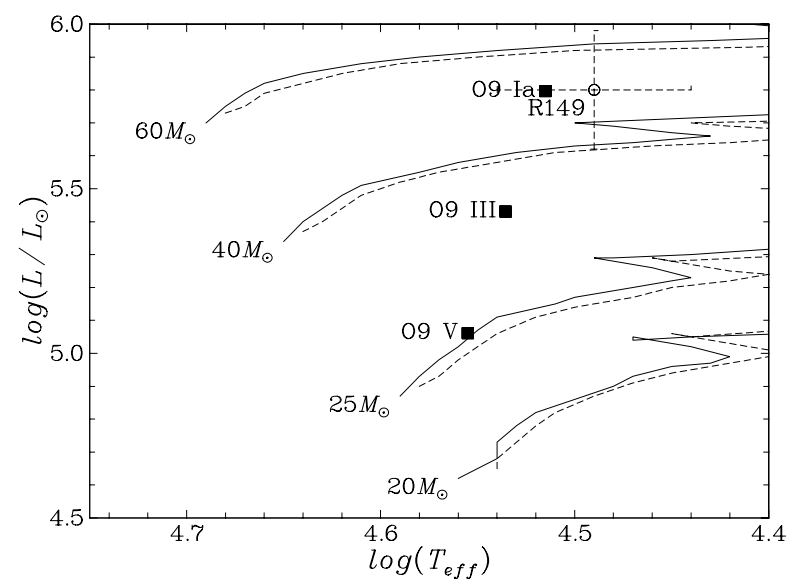

Fig. 7. Evolution tracks for several masses, plotted from the Geneva grid of models (Lejeune \& Schaerer 2001). Solid lines: LMC metallicity, $Z=0.008$; dashed lines: Galactic metallicity, $Z=0.02$. Filled squares represent the positions of $\mathrm{O} 9$ type stars of different luminosity class (Vacca et al. 1996). Open circle shows the position of \#193 (R 149), assuming $M_{V}$ between -6.8 and $-6.6, T_{\text {eff }}$ between 27500 and $34300 \mathrm{~K}$, and a bolometric correction between -3.4 and -2.7 (Vacca et al. 1996).

not exactly sample the same zones. Anyhow, the higher values are supported by our HST (Paper I) and present ISAAC observations. 


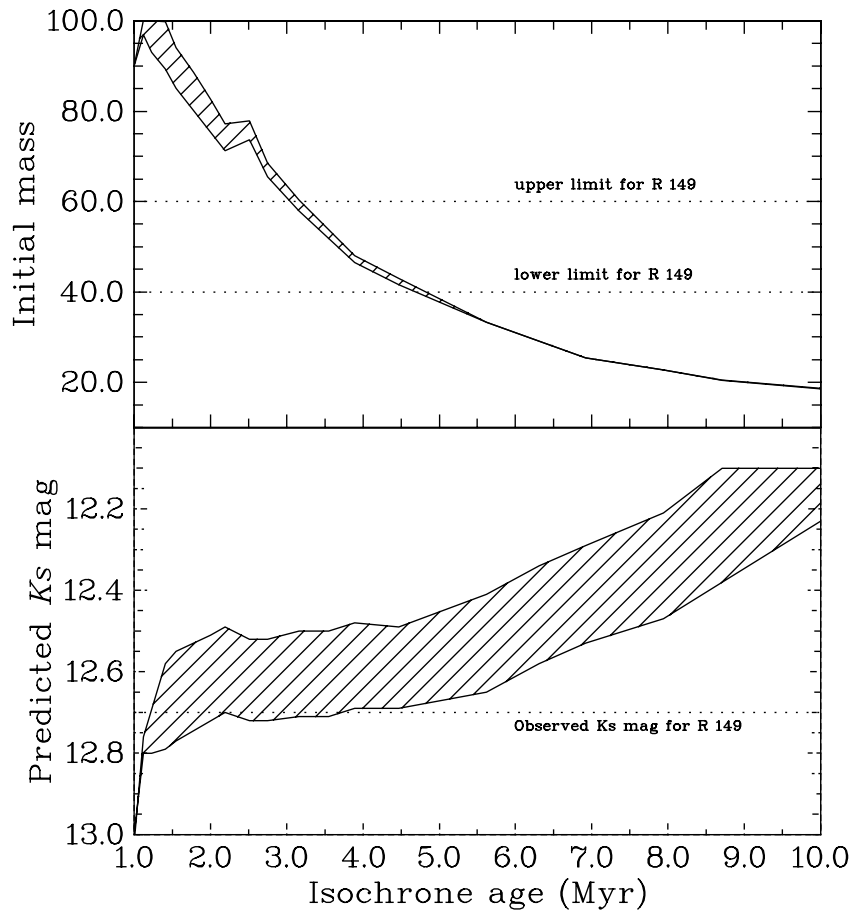

Fig. 8. Expected initial mass (top) and $K$ s magnitude (bottom) for stars with $M_{V}$ between -6.8 and -6.6 mag as a function of age. The data is taken from the Geneva grid of models for $Z=0.008$ (Lejeune $\&$ Schaerer 2001). Shaded areas correspond to points with $M_{V}$ between -6.8 and -6.6 . Top: The mass range deduced from Fig. 7 is represented by two horizontal dotted line at $M=60 M_{\odot}$ and $M=40 M_{\odot}$. Bottom: The observed $K$ s magnitude is represented by a horizontal dotted line at $K \mathrm{~s}=12.63 \mathrm{mag}$.

\section{Discussion}

The population of young massive stars, which was discussed earlier (Sect. 3.2), is spatially distributed over the whole field, while a sample of it, represented by triangles in Figs. 5 and 6, is grouped in a cluster, marked as region B. This grouping is expected given the young age of these stars. How can though one explain the separation between this cluster and the other massive stars which even if they have similar ages are at a considerable distances from the cluster, for example $\sim 70^{\prime \prime}(\sim 18 \mathrm{pc})$ for star \#193, one of the most distant? One explanation could be that massive star formation may have taken place simultaneously at different parts of the molecular cloud. At these locations the molecular material has been fully dissociated and ionized, and we do not observe it now. Alternatively, massive star formation has occurred in cluster $\mathrm{B}$, and subsequently a number of the members have been ejected due to the dynamics of the cluster.

It has been shown that once an embedded cluster forms, three mass evacuation mechanisms work over different timescales to disrupt it (Kroupa 2001): a) expulsion of embryonic gas (approximately during the first 0-5 Myr), b) mass loss from evolving stars (significant after $\sim 3 \mathrm{Myr}$ ), and c) stellar dynamical evaporation and ejection of stars (all times). Binary-binary collisions are required to produce high velocity escapees to occur in low density clusters
(Leonard \& Duncan 1988, 1990), although simple calculations suggest that such interactions are rather unlikely. Recently Vine \& Bonnell (2003) have studied the dynamics of massive stars in young clusters containing gas and stars. They have shown that the location of massive stars outside the core of the cluster does not exclude their formation in the dense cluster core. The massive stars could have originated in the core, but escaped from that region during the gas expulsion phase. Furthermore, the ejection of the $\mathrm{OB}$ stars must have happened during an earlier evolutionary stage when the cluster was most probably more compact than today (Portegies Zwart et al. 1999). Assuming that star \#193 has been kicked out of cluster B, an escape velocity of $\sim 5.5 \mathrm{~km} \mathrm{~s}^{-1}$ has been necessary for it to reach its observed position after a travel time of 3 Myr. This estimate is a lower limit due to projection on the sky of a threedimensional configuration in space. Higher velocities are quite possible since escapees can leave their birthplace with velocities up to $100 \mathrm{~km} \mathrm{~s}^{-1}$ or even larger (Leonard \& Duncan 1990; Kroupa 1995).

We note also that all the candidate massive stars are devoid of proper nebulosity, in contrast to the Papillon. This fact suggests that the Papillon is probably the youngest visible massive star formation event in the whole field. The strength of the molecular hydrogen emission detected towards the Papillon confirms its nature as a very young star formation region (Israel \& Koornneef 1988; Kawara et al. 1988). In fact the observed luminosity of the $\mathrm{H}_{2}$ line $v=1-O S(1)$ towards the Papillon is two times larger than that observed at the Orion source (Kawara et al. 1988). The massive star(s) powering the Papillon have not had enough time to disrupt the H II region. Moreover, the presence of nebulosity excludes the possibility for the Papillon of ejection from cluster B. It is therefore conceivable that the Papillon lies somewhat above or below the mean plane of N 159. We believe that the Papillon is situated at the side nearer to us since it is visible in the optical. We estimate that star \#371, which lies towards the center of the Papillon, has a mass of $\sim 50 M_{\odot}$, even though based on our current resolution we cannot exclude the possibility that the star is multiple. Should other massive stars be embedded inside that nebula, much better spatial resolution and deeper exposures are required in order to uncover them. From our previous $H S T$ observations we estimated an exciting star of type at least $\mathrm{O} 8 \mathrm{~V}$, $\sim 30 M_{\odot}$ (Vacca et al. 1996), for the Papillon using the $\mathrm{H} \beta$ flux measurement and assuming that the H II region is ionization bounded (Paper I). The difference between the two mass estimates is due to the fact the H II region is density-bounded at least towards us and that the flux correction for extinction is not straightforward. The latter point is probably the reason why the radio continuum observations, which are less affected by extinction, yield a higher Lyman continuum flux. In an earlier work (Heydari-Malayeri \& Testor 1985), we used the radio continuum observations at $843 \mathrm{MHz}$, obtained with a beam of $43^{\prime \prime} \times 46^{\prime \prime}$, to derive a flux density of $55 \mathrm{mJy}$ for $\mathrm{N} 159-5$, after correcting for contamination by the surrounding field. A resulting ultraviolet flux of $\sim 1.2 \times 10^{49}$ photons indicates an O7 V type star of $\sim 38 M_{\odot}$ (Vacca et al. 1996). Given the uncertainties involved, the stellar mass estimates based on the 


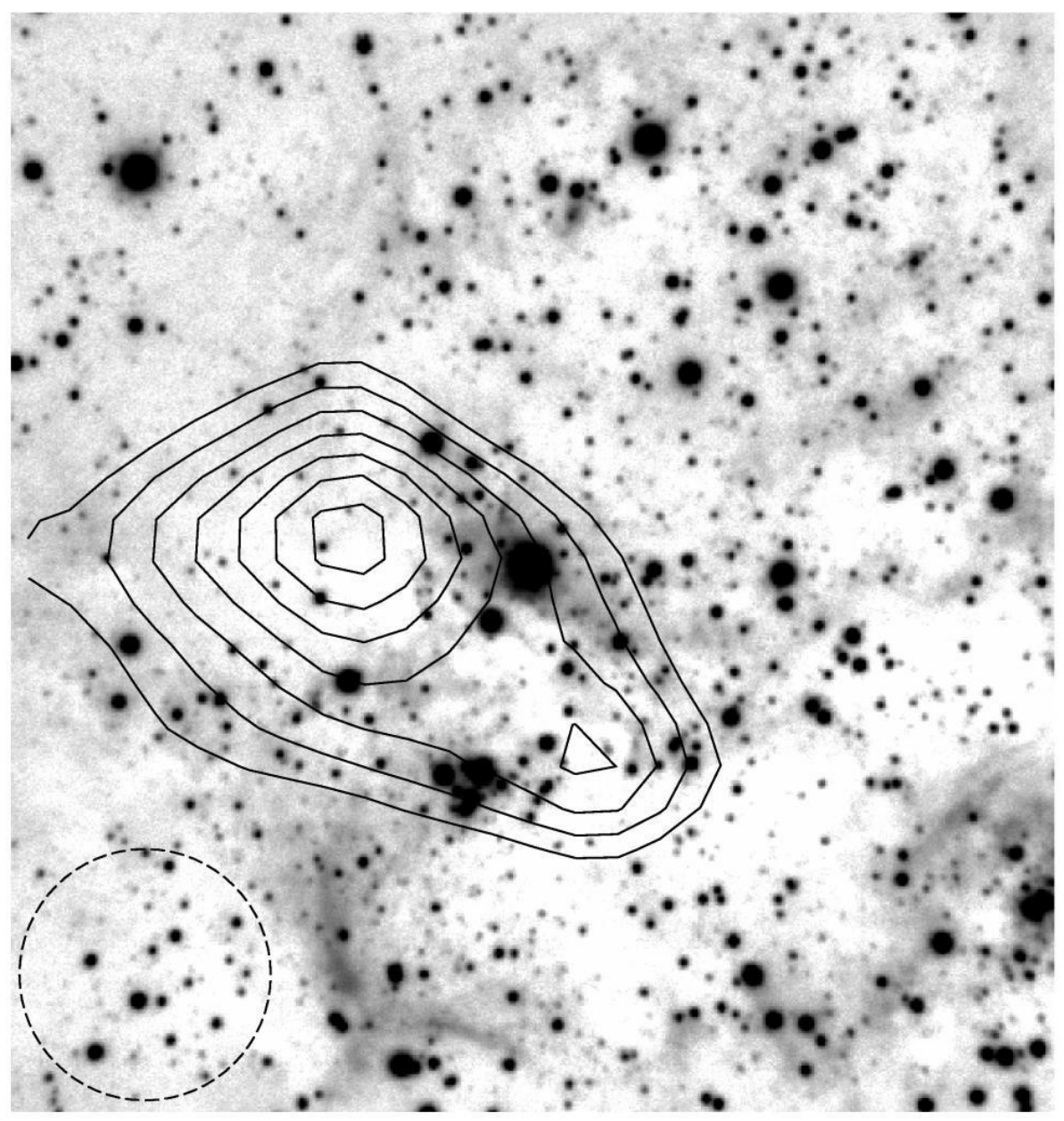

Fig. 9. An $H$ band image of our field with a contour overlay of the ${ }^{12} \mathrm{CO}(1-0)$ intensity of the molecular cloud N159-East from Johansson et al. (1998). The field size and orientation are the same as in Fig. 1, and the SEST $\sim 40^{\prime \prime}{ }^{12} \mathrm{CO}(1-0)$ beam is marked with a dotted circle.

H I emission from the nebula agree well with the $\sim 50 M_{\odot}$ derived from photometry using evolutionary models.

An age of $\sim 3$ Myr was derived for the massive star population using the evolutionary models and supplementary data on one of the members. We wish to note though that this age estimate may not be very accurate due to the degeneracy of the near IR colors of massive stars. In fact any isochrone between 1 and 10 Myrs would be consistent with our data. We favored the $3 \mathrm{Myr}$ isochrone in order to meet the requirements of star Sk-69 257 .

One can also estimate the number of stars which power the H II region N 159 on the basis of radio continuum observations. Clarke et al. (1976) measured a radio continuum flux density of $6.5 \mathrm{Jy}$ at $408 \mathrm{MHz}$ using the Molonglo telescope whose beam had a width of $2.6 \times 2 ! 9$. The beamwidth is comparable with the size of our ISAAC field, and the target coordinates match the position of the Papillon, while the reported pointing accuracy is $18^{\prime \prime} \times 5^{\prime \prime}$. The derived Lyman continuum flux of $1.36 \times 10^{51}$ photons $\mathrm{s}^{-1}$ corresponds to some 40 massive stars of type $\mathrm{O} 5 \mathrm{~V}$ with an initial mass of $\sim 50 M_{\odot}$ (Vacca et al. 1996). Taking a Salpeter-like initial mass function with slope $x=-1.5$, we can predict the presence of some 360 stars of mass about $10 M_{\odot}$ and 3240 stars of $\sim 2 M_{\odot}$. Where are these
$40 \mathrm{O} 5 \mathrm{~V}$ stars? It is quite possible that they are among the stars we imaged but due to the degeneracies in the colors mentioned earlier they can only be clearly identified if spectroscopic observations were available. Moreover, some of them may be embedded in the molecular cloud and some situated outside our ISAAC field.

The color-mag diagram (Fig. 5) also shows the presence of intermediate mass stars of $\sim 4-10 M_{\odot}$ on the main sequence formed together with high mass stars $\sim 3$ Myr ago. This is in agreement with more detailed results on the Orion Nebular Cluster (ONC) based on a large body of data $(\sim 3500$ stars identified within $\sim 2.5 \mathrm{pc}$ of the Trapezium, among which at least $\sim 1600$ with photometric and spectroscopic data in the visible) (Hillenbrand 1997). According to these studies, low-, intermediate-, and high-mass stars have formed together in the ONC a few Myr ago (Palla \& Stahler 1999). However, this may not be a universal trend since Herbst \& Miller (1982)'s study of NGC 3293 led them to the conclusion that in a cluster low- and intermediate-mass stars form first, with the process continuing gradually until the high-mass stars appear. This result is in agreement with more recent findings on star formation in LMC clusters and associations. For instance, in the case of the R 136 cluster, situated in the LMC 30 Dor, 
Massey \& Hunter (1998) arrived to the conclusion that intermediate-mass stars began forming some $6 \mathrm{Myr}$ ago and continued up to the time when the high-mass stars formed, 12 Myr ago.

An interesting question is whether the young ( $3 \mathrm{Myr})$ and old ( $\sim 1-10$ Gy) stellar populations have formed in the same region of space. Although presently we do not have the necessary data to address this issue and cannot reach a firm conclusion, it is quite possible that both populations be spatially unrelated. The LMC is known to have a considerable depth, the old population can have formed in a different depth during much earlier star formation activities. In order to get some insight about this question, we used the 2MASS data to probe a bare stellar field devoid of any particular nebular emission lying near the $\mathrm{N} 159$ complex (radius 1.22 , centered on $\alpha=05 \mathrm{~h} 39 \mathrm{~m} 00 \mathrm{~s}$, $\left.\delta=-69^{\circ} 47^{\prime} 30^{\prime \prime}\right)$. The corresponding HR diagram shows the absence of a young, unreddened population, but the presence of an old population resembling the one found towards N 159. Although this population is relatively smaller in number with respect to that of $\mathrm{N} 159$, since 2MASS is not as deep as our photometry, the presence of the old population is certain. The old population seems therefore to be a common background stellar component towards this part of the LMC.

The presence of low-mass pre-main sequence LMC stars in the above diagrams seems unlikely, even if those objects are characterized by large near IR colors, $H-K \sim 1.5 \mathrm{mag}$ (Lada \& Adams 1992; Chabrier et al. 2000). A pre-main sequence star of $\sim 1 M_{\odot}$ has a luminosity of $\log \left(L / L_{\odot}\right) \sim 1$ on its birthline, corresponding to an observed visual magnitude of $\sim 21$, which is below our detection limit. An intermediate mass pre-main sequence star of $5 M_{\odot}$ has an effective temperature of $\sim 11000 \mathrm{~K}$ and $\log \left(L / L_{\odot}\right) \sim 3$, corresponding to a reddened 16 mag star, occupying loci around $J-H \sim 0.5$ and $H-K=0.5$ mag (Lada $\&$ Adams 1992). There is a few number of sources with such colors in Fig. 6, given the color uncertainties at those magnitudes. Therefore, we cannot exclude the possibility that some of those points represent intermediate mass PMS stars. As for more massive objects, the concept of pre-main sequence is not applicable to stars above $\sim 6 M_{\odot}$ since the birthline and the ZAMS unify at those mass levels (Palla \& Stahler 1993).

Comparison between LMC N 159 and SMC N 81 points out dramatic differences between the environments of these two HEBs. The present work shows the Papillon as part of a rich complex containing a large molecular cloud and a cluster of young, massive stars, whereas our previous study of SMC N 81, based on ISAAC near IR observations (Heydari-Malayeri et al. 2003), revealed a solitary star formation event. Moreover, since the two compact H II regions have several comparable characteristics, if we assume that they have gone through a similar formation process, then the HEB formation can take place in both very dense as well as rather sparse environments.

Acknowledgements. We are grateful to Dr. L. E. B. Johansson for providing us with the CO map of the N 159 molecular cloud. V.C. would like to acknowledge the support of JPL contract 960803 . F.M. wishes to thank Dr. Eric Mandel for his valuable help concerning the DS9 astronomical data visualization application (Joye \& Mandel 2003). We would like also to thank the referee, Dr. Joao Alves, for useful advices. Finally, this publication makes use of data products from the Two Micron All Sky Survey, which is a joint project of the University of Massachusetts and the Infrared Processing and Analysis Center/California Institute of Technology, funded by the National Aeronautics and Space Administration and the National Science Foundation.

\section{References}

Bolatto, A. D., Jackson, J. M., Israel, F. P., Zhang, X., \& Kim, S. 2000, ApJ, 545, 234

Brooks, K. J., \& Whiteoak, J. B. 1997, MNRAS, 291, 395

Carpenter, J. M. 2001, AJ, 121, 2851

Chabrier, G., Baraffe, I., Allard, F., \& Hauschildt, P. 2000, ApJ, 542, 464

Clarke, J. N., Little, A. G., \& Mills, B. Y. 1976, Aust. J. Phys. Astrophys. Suppl., 40, 1

Clayton, G. C., \& Martin, P. G. 1985, ApJ, 288, 558

Conti, P. S., Garmany, C. D., \& Massey, P. 1986, AJ, 92, 48

Crowther, P. A., Hillier, D. J., Evans, C. J., et al. 2002, ApJ, 579, 774

Cutri, R. M., Skrutskie, M. F., van Dyk, S., et al. 2003a, VizieR Online Data Catalog, 2246, 0

Cutri, R. M., Skrutskie, M. F., van Dyk, S., et al. 2003b, Explanatory Supplement to the 2MASS All Sky Data Release (Pasadena: Caltech), www.ipac.caltech.edu/2mass/ releases/allsky/doc/

Davies, R., Eliott, K., \& Meaburn, J. 1976, Mem. R. Astron. Soc., 81, 89

Deharveng, L., Caplan, J., \& Lombard, J. 1992, A\&AS, 94, 359

Deharveng, L., Nadeau, D., Zavagno, A., \& Caplan, J. 2000, A\&A, 360,1107

Dickey, J. M., Mebold, U., Marx, M., et al. 1994, A\&A, 289, 357

Dufour, R. J., \& Duval, J. E. 1975, PASP, 87, 769

Heikkilä, A., Johansson, L. E. B., \& Olofsson, H. 1999, A\&A, 344, 817

Henize, K. G. 1956, ApJS, 2, 315

Herbst, W., \& Miller, D. P. 1982, AJ, 87, 1478

Herrero, A., Puls, J., \& Najarro, F. 2002, A\&A, 396, 949

Heydari-Malayeri, M., \& Testor, G. 1982, A\&A, 111, L11

Heydari-Malayeri, M., \& Testor, G. 1985, A\&A, 144, 98

Heydari-Malayeri, M., Charmandaris, V., Deharveng, L., Rosa, M. R., \& Zinnecker, H. 1999a, A\&A, 347, 841

Heydari-Malayeri, M., Rosa, M. R., Charmandaris, V., Deharveng, L., \& Zinnecker, H. 1999b, A\&A, 352, 665, Paper I

Heydari-Malayeri, M., Rosa, M. R., Zinnecker, H., Deharveng, L., \& Charmandaris, V. 1999c, A\&A, 344, 848

Heydari-Malayeri, M., Charmandaris, V., Deharveng, L., et al. 2001a, A\&A, 372, 527

Heydari-Malayeri, M., Charmandaris, V., Deharveng, L., et al. 2001b, A\&A, 372, 495

Heydari-Malayeri, M., Charmandaris, V., Deharveng, L., et al. 2002a, A\&A, 381, 941

Heydari-Malayeri, M., Rosa, M. R., Schaerer, D., Martins, F., \& Charmandaris, V. 2002b, A\&A, 381, 951

Heydari-Malayeri, M., Meynadier, F., Charmandaris, V., et al. 2003, A\&A, 411, 427

Hillenbrand, L. A. 1997, AJ, 113, 1733

Israel, F. P., \& Koornneef, J. 1988, A\&A, 190, 21

Israel, F. P., Maloney, P. R., Geis, N., et al. 1996, ApJ, 465, 738

Johansson, L. E. B., Greve, A., Booth, R. S., et al. 1998, A\&A, 331, 857

Jones, T. J., Hyland, A. R., Straw, S., et al. 1986, MNRAS, 219, 603 
Joye, W. A., \& Mandel, E. 2003, in Astronomical Data Analysis Software and Systems XII, ASP Conf. Ser., 295, 489

Kawara, K., Taniguchi, Y., \& Nishida, M. 1988, PASP, 100, 458

Kroupa, P. 1995, MNRAS, 277, 1522

Kroupa, P. 2001, in From Darkness to Light: Origin and Evolution of Young Stellar Clusters, ASP Conf. Ser., 243, 387

Lada, C. J., \& Adams, F. C. 1992, ApJ, 393, 278

Le Bertre, T., Matsuura, M., Winters, J. M., et al. 2001, A\&A, 376, 997

Lejeune, T., \& Schaerer, D. 2001, A\&A, 366, 538

Leonard, P. J. T., \& Duncan, M. J. 1988, AJ, 96, 222

Leonard, P. J. T., \& Duncan, M. J. 1990, AJ, 99, 608

Lequeux, J. 1989, in Recent Developments of Magellanic Cloud Research. A European Coll., held in Paris, France, May 9-11, ed. K. S. de Boer, F. Spite, \& G. Stasinska (Meudon, France: Observatoire de Paris, Section Astrophysique de Meudon), 119

Lucke, P. B., \& Hodge, P. W. 1970, AJ, 75, 171

Markova, N., Puls, J., Repolust, T., \& Markov, H. 2004, A\&A, accepted

Martins, F., Schaerer, D., \& Hillier, D. J. 2002, A\&A, 382, 999
Massey, P., \& Hunter, D. A. 1998, ApJ, 493, 180

McGee, R. X., Brooks, J. W., \& Batchelor, R. A. 1972, Aust. J. Phys., 25,581

Mills, B. Y., \& Turtle, A. J. 1984, in Structure and Evolution of the Magellanic Clouds, IAU Symp., 108, 283

Nandy, K., Morgan, D. H., Willis, A. J., Wilson, R., \& Gondhalekar, P. M. 1981, MNRAS, 196, 955

Palla, F., \& Stahler, S. W. 1993, ApJ, 418, 414

Palla, F., \& Stahler, S. W. 1999, ApJ, 525, 772

Portegies Zwart, S. F., Makino, J., McMillan, S. L. W., \& Hut, P. 1999, A\&A, 348, 117

Schmidt-Kaler, T., Gochermann, J., Oestreicher, M. O., et al. 1999, MNRAS, 306, 279

Stetson, P. B. 1987, PASP, 99, 191

Vacca, W. D., Garmany, C. D., \& Shull, J. M. 1996, ApJ, 460, 914

van Loon, J. T. 2000, A\&A, 354, 125

van Loon, J. T., Groenewegen, M. A. T., de Koter, A., et al. 1999, A\&A, 351, 559

Vine, S. G., \& Bonnell, I. A. 2003, MNRAS, 342, 314

Walborn, N. R. 1977, ApJ, 215, 53 